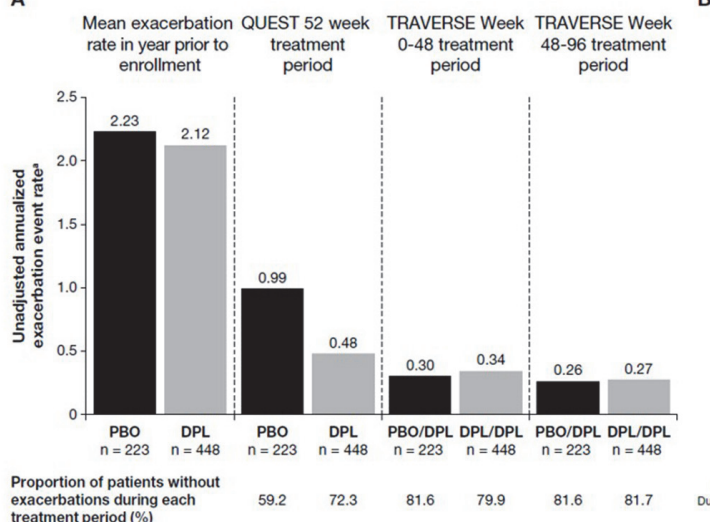

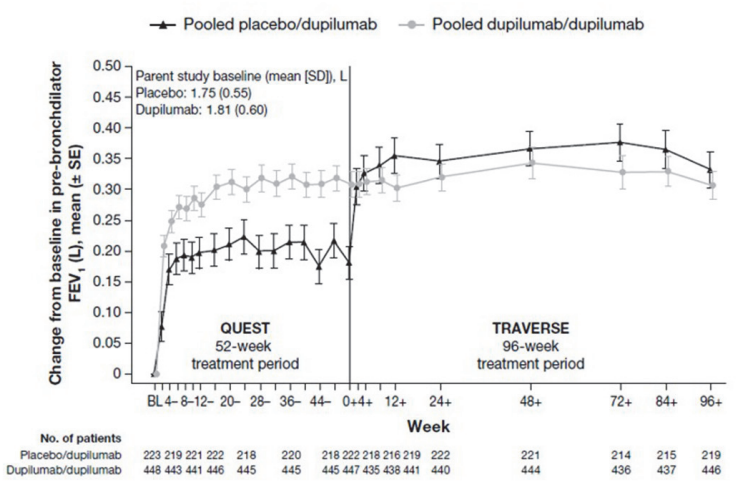

Abstract S84 Figure 1 Efficacy endpoints in QUEST patients who rolled over into TRAVERSE completing 3 full years of dupilumab treatment. (A) Unadjusted; (B) Change from QUEST baseline in FEV 1

(A) AER analysis in blinded parent study was done on ITT and in the open label TRAVERSE was done on the safety population.

(B) FEV 1 was assessed in the exposed population (observed cases) using descriptive statistics. BL represents the baseline of the parent study. Week 0 represents the start of TRAVERSE, and Week in TRAVERSE refers to the time in TRAVERSE without regard to any time in any parent study.

${ }^{a}$ The total number of events that occurred during the treatment period divided by the total number of patient-years followed in the treatment period. $\mathrm{BL}$, baseline; ITT, intention-to-treat; SD, standard deviation; SE, standard error.

$300 \mathrm{mg}$ q2w. We assessed the unadjusted annualized rate of severe asthma exacerbations (AER) during QUEST (Weeks 052) and TRAVERSE (Weeks $0-48$ and Weeks 48-96) and the mean change from QUEST baseline in pre-bronchodilator $\mathrm{FEV}_{1}$ in QUEST over the QUEST and TRAVERSE studies.

Results The unadjusted AER in dupilumab-treated patients was low during QUEST, with progressive reductions observed during the treatment period of 96 weeks in TRAVERSE and a majority of patients experiencing no exacerbations (figure 1A). In the placebo/dupilumab group of patients, the unadjusted AER decreased significantly during TRAVERSE compared with QUEST and was similar to the AER in dupilumab/dupilumab patients. Clinically meaningful improvements in pre-bronchodilator $\mathrm{FEV}_{1}$ were observed at Week 52 of QUEST in dupilumab/dupilumab patients, which were sustained with no apparent loss of treatment effect at Weeks 48 and 96 of TRAVERSE (figure 1B). Large improvements in pre-bronchodilator $\mathrm{FEV}_{1}$ were observed in placebo/dupilumab patients upon initiation of dupilumab, which were sustained throughout the TRAVERSE treatment period.

Conclusions Dupilumab demonstrated sustained efficacy in reducing severe asthma exacerbations and improving lung function in patients with moderate-to-severe asthma who completed 96 weeks of treatment.

Please refer to page A189 for declarations of interest related to this abstract.

\section{S85 CLINICAL CHARACTERISTICS ASSOCIATED WITH MUCUS PLUGGING IN SEVERE EOSINOPHILIC ASTHMA AND THE EFFECTIVENESS OF BENRALIZUMAB TREATMENT}

${ }^{1}$ AP Hearn, ${ }^{2}$ MS Mak, ${ }^{2}$ I Budaj, ${ }^{3} \mathrm{~N}$ Qurashi, ${ }^{3} \mathrm{O}$ Snell, ${ }^{1} \mathrm{~J}$ Kavanagh, ${ }^{1} \mathrm{M}$ Fernandes, 'L Green, ${ }^{1} \mathrm{C}$ Roxas, , 'L Thomson, ' $\mathrm{G}$ d'Ancona, '1 Dhariwal, 'AM Nanzer, 'DJ Jackson. 'Guy's Severe Asthma Centre, UK, London; ' Guy's and St Thomas Department of Radiology, London, UK; ${ }^{3}$ King's College London, London, UK
Background Eosinophil-rich mucus plugs are a recognised feature of asthma and are believed to contribute to airflow obstruction. However, little is known about the incidence of plugging in severe eosinophilic asthma (SEA) and the relationship of plugging to other characteristics of this phenotype. In addition, it is unknown whether patients with evidence of plugging have a differential response to the eosinophil depleting anti-IL-5 R therapy benralizumab.

Methods Pre-biologic CT scans of patients with SEA treated with benralizumab were assessed by two radiologists independently. Patients were given a mucus score out of 20 (per segment plugged; score of 1-3 classed as plugging-low, score ${ }^{3} 4$ as plugging-high). Baseline characteristics and clinical outcome following 1 year of treatment was compared between patients with and without evidence of plugging.

Results CT scans of 116 patients with SEA were reviewed. 69/ 116 (59.5\%) exhibited mucus plugging of which 40/116 $(34.5 \%)$ were classed as plugging-high. Median (IQR) number of segments plugged was 4 (2-10.5). Baseline characteristics were similar between the plugging and no-plugging groups, with a trend towards lower baseline FEV1 in the plugging group $(p=0.06)$. Nasal polyposis was more frequent in the plugging-high group (45\%) compared to plugging low (24\%) and unplugged $(21 \%)$ groups (ANOVA $\mathrm{p}=0.04$ ). Response to benralizumab at 1 year was comparable between those with and without plugging: AER reduction $3.67+/-4.1$ vs $3.17+/$ 3.47; change in ACQ6 $0.73+/-1.1$ vs $0.77+/-1.38$; change in FEV1\% $6.72+/-17.35$ vs $6.50+/-18.3$ (all $\mathrm{p}=\mathrm{NS}$ ). The number of plugged segments at baseline did not correlate with improvement in FEV1.

Discussion Mucus plugging is identified in $60 \%$ of patients with SEA and is more commonly seen at high levels in patients with co-morbid nasal polyposis. Individuals with SEA and mucus plugging have a similar clinical response to eosinophil targeted therapy with benralizumab compared to patients with SEA but absent plugging on CT imaging. 
Abstract S85 Table 1 Baseline Characteristics

\begin{tabular}{|c|c|c|c|c|c|c|c|}
\hline & $\begin{array}{l}\text { Whole cohort } \\
n=116\end{array}$ & Plugged $n=69$ & $\begin{array}{l}\text { No Plugging } \\
n=47\end{array}$ & $\begin{array}{l}\text { Plugging low } \\
n=29\end{array}$ & $\begin{array}{l}\text { Plugging High } \\
n=40\end{array}$ & $\begin{array}{l}\text { P value plugged } \\
\text { vs unplugged }\end{array}$ & $\begin{array}{l}\text { P value across } \\
\text { plugging groups }\end{array}$ \\
\hline Female & 73 & 44 & 29 & 14 & 30 & 0.82 & 0.07 \\
\hline $\mathrm{N}(\%)$ & (62.9) & (63.8) & $(61.7)$ & $(48.3)$ & (75) & & \\
\hline Age & 52.3 & 53.94 & 49.96 & 55 & 53.18 & 0.10 & 0.22 \\
\hline Mean (SD) & (12.8) & (13.2) & (11.8) & (12.9) & (13.6) & & \\
\hline BMI & 31.3 & 31.0 & 31.7 & 30.5 & 31.3 & 0.59 & 0.78 \\
\hline Mean (SD) & $(7.2)$ & (6.6) & (8.0) & (6.4) & (6.7) & & \\
\hline AER & 4.8 & 4.7 & 4.9 & 4.6 & 4.8 & 0.67 & 0.83 \\
\hline Mean (SD) & (3.3) & (3.0) & (3.9) & (3.0) & (3.0) & & \\
\hline mOCS & 64 & 39 & 25 & 17 & 22 & 0.72 & 0.90 \\
\hline $\mathrm{N}(\%)$ & $(55.2)$ & $(56.5)$ & $(53.2)$ & (58.6) & $(55.0)$ & & \\
\hline Peak Eosinophil & 0.6 & 0.6 & 0.6 & 0.6 & 0.7 & 0.40 & 0.45 \\
\hline Count & $(0.4-0.9)$ & $(0.5-0.9)$ & $(0.4-0.8)$ & $(0.5-0.7)$ & $(0.5-1.0)$ & & \\
\hline \multicolumn{8}{|l|}{ Median (IQR) } \\
\hline FENO ppb & 44 & 44 & 47 & 62 & 38.5 & 0.74 & 0.3 \\
\hline Median (IQR) & $(25-78)$ & $(27-83)$ & $(23-67)$ & $(26-93)$ & $(27.5-58.5)$ & & \\
\hline Adult onset & 59 & 37 & 22 & 15 & 22 & 0.47 & 0.74 \\
\hline N (\%) & $(50.9)$ & $(53.6)$ & $(46.8)$ & $(51.7)$ & $(55.0)$ & & \\
\hline Atopy & 86 & 47 & 39 & 18 & 29 & 0.07 & 0.12 \\
\hline $\mathrm{N}(\%)$ & $(74.1)$ & $(68.1)$ & $(83.0)$ & $(62.1)$ & $(72.5)$ & & \\
\hline Nasal Polyps & 35 & 25 & 10 & 7 & 18 & 0.08 & 0.04 \\
\hline $\mathrm{N}(\%)$ & $(30.2)$ & $(36.2)$ & $(21.3)$ & (24.1) & $(45.0)$ & & \\
\hline FEV1 Litres & 1.7 & 1.6 & 1.86 & 1.6 & 1.6 & 0.06 & 0.17 \\
\hline Mean (SD) & $(0.7)$ & $(0.6)$ & $(0.7)$ & $(0.7)$ & $(0.7)$ & & \\
\hline FEV $1 \%$ Predicted & 61.7 & 59.6 & 64.8 & 58.5 & 60.4 & 0.20 & 0.42 \\
\hline Mean (SD) & $(21.6)$ & (19.8) & $(30.0)$ & (21.3) & $(18.9)$ & & \\
\hline ACQ-6 & 2.9 & 2.8 & 3.2 & 2.9 & 2.7 & 0.12 & 0.26 \\
\hline Mean (SD) & (1.4) & (1.4) & (1.3) & (1.4) & (1.4) & & \\
\hline Mini-AQLQ & 3.3 & 3.3 & 3.2 & 3.0 & 3.6 & 0.68 & 0.25 \\
\hline Mean (SD) & (1.7) & (1.7) & (1.8) & (1.8) & (1.5) & & \\
\hline Non-Smokers & 77 & 42 & 35 & 15 & 27 & 0.19 & 0.24 \\
\hline N (\%) & $(66.4)$ & $(60.9)$ & $(74.5)$ & (15.7) & $(67.5)$ & & \\
\hline Ex-Smokers & 37 & 26 & 11 & 14 & 12 & & \\
\hline N (\%) & (31.9) & (37.7) & $(23.4)$ & $(48.3)$ & (30) & & \\
\hline Current Smokers & 1 & 1 & 0 & 0 & 1 & & \\
\hline $\mathrm{N}(\%)$ & $(0.9)$ & (1.4) & & & (2.5) & & \\
\hline
\end{tabular}

For normally distributed data, number quoted is mean (standard deviation). For non-parametric variables, number quoted is median (inter-quartile range).

Abbreviations: ACQ6 = Asthma Control Questionnaire 6; Receptor; BMI = Body Mass Index; FeNO = Fractional exhaled Nitric Oxide; FEV1 = Forced Expiratory Volume in 1 second; mOCS = maintenance Oral Corticosteroid; Mini-AQLQ = Mini Asthma Quality of Life Questionnaire; $p p b=$ parts per billion;

\section{S86 LONG-TERM ASSESSMENT OF EXACERBATIONS AND LUNG FUNCTION IN THE LIBERTY ASTHMA TRAVERSE STUDY, STRATIFIED BY LUNG FUNCTION IMPROVEMENTS AT THE END OF THE PHASE 3 LIBERTY ASTHMA QUEST PARENT STUDY}

${ }^{1} \mathrm{~A}$ Bourdin, ${ }^{2} \mathrm{NA}$ Hanania, ${ }^{3} \mathrm{D}$ Dorscheid, ${ }^{4} \mathrm{X}$ Muñoz, ${ }^{5} \mathrm{Y}$ Tohda, ${ }^{6} \mathrm{~N}$ Daizadeh, ${ }^{7} \mathrm{~J}$ Jacob-Nara, ${ }^{8} \mathrm{~B}$ Ortiz, ${ }^{6} \mathrm{M}$ Djandji, ${ }^{8} \mathrm{Y}$ Deniz, ${ }^{7} \mathrm{PJ}$ Rowe. ${ }^{1}$ University of Montpellier, Montpellier, France; ${ }^{2}$ Baylor College of Medicine, Houston, TX, USA; ${ }^{3}$ Center for Heart Lung Innovation, University of British Columbia, Vancouver, BC, Canada; ${ }^{4}$ Hospital Vall d'Hebron, Barcelona, Spain; ${ }^{5}$ Kindai University Hospital, Osakasayama, Osaka, Japan; ${ }^{6}$ Sanofi, Cambridge, MA, USA; ${ }^{7}$ Sanofi, Bridgewater, NJ, USA; ${ }^{8}$ Regeneron Pharmaceuticals, Inc., Tarnytown, NY, USA

\subsection{6/thorax-2021-BTSabstracts.92}

Introduction and Objectives Low pre-bronchodilator $\mathrm{FEV}_{1}$ in asthma patients is associated with increased exacerbations and lung function decline. Dupilumab, a fully human monoclonal antibody, blocks the shared receptor component for interleukin-4 and interleukin-13, key and central drivers of type 2 inflammation in multiple diseases. TRAVERSE (NCT02134028), a single-arm, open-label extension study, evaluated long-term safety, tolerability, and efficacy of dupilumab added to standard of care in adult/adolescent patients continuing from a previous dupilumab asthma study. Post-hoc analysis of TRAVERSE patients enrolled from QUEST (NCT02414854) with baseline blood eosinophils $\geq 150$ cells $/ \mu \mathrm{L}$ or FeNO $\geq 25$ parts per billion assessed the relationship of lung function (characterized by baseline and change in pre-bronchodilator $\mathrm{FEV}_{1}$ and $\mathrm{FEV}_{1}$ : forced vital capacity [FVC] ratio) on severe exacerbation rate (AER).

Methods Patients with increased type 2 inflammatory biomarkers who participated in QUEST (52 weeks) and continued to TRAVERSE (up to 96 weeks) were stratified by prebronchodilator $\mathrm{FEV}_{1}$ improvement at Week 52 of QUEST. Endpoints assessed were exacerbation history in the year before QUEST; annualized AER during QUEST and 